\title{
COOPERATIVITY AMONG AFFERENTS FOR THE INDUCTION OF LONG-TERM POTENTIATION IN THE CA1 REGION OF THE HIPPOCAMPUS
}

\author{
KEVIN S. LEE \\ Nerve Cell Biology Unit, Department of Neuromorphology, Max Planck Institute for Psychiatry, Kraepelinstrasse 2, 8000 \\ Munich 40, Federal Republic of Germany
}

Received October 19, 1982; Revised January 10, 1983; Accepted January 18, 1983

\begin{abstract}
Long-term potentiation of synaptic responses in the hippocampus is a unique form of physiological plasticity which can be induced by brief episodes of repetitive afferent activity and which can persist for days or months. The present study describes cooperative (or associative) interactions among afferents to the stratum radiatum of CA1 for the induction and maintenance of this phenomenon. A greater degree of long-term potentiation was obtained when adjacent afferents to the stratum radiatum were co-actively conditioned, as compared to that observed with activation of a single pathway alone. This cooperativity was not the result of increased postsynaptic discharge during conditioning, as shown by the absence of greater long-term potentiation following orthodromic and antidromic co-activation of the postsynaptic CA1 pyramidal cells.
\end{abstract}

Sustained modification of the efficacy of synaptic connections following brief, high frequency episodes of activity has been described in several systems associated with the hippocampus (Bliss and Lomo, 1973; Bliss and Gardner-Medwin, 1973; Douglas and Goddard, 1975; Schwartzkroin and Wester, 1975; Alger and Teyler, 1976) and recently in the cerebral cortex (Lee, 1982). This change has been termed long-term potentiation or, more recently, enhancement (McNaughton et al., 1978; McNaughton, 1982) to distinguish it from the shorter-term phenomenon of post-tetanic potentiation. This capacity for change is often discussed in the context of learning and memory inasmuch as it can be induced by rather brief events and has been shown to persist for weeks or months, thus providing an attractive substrate for sustained behavioral modification. Recent evidence from input systems to the dentate gyrus suggests that an interaction or cooperativity exists among entorhinal cortical afferents for the induction of this phenomenon. Specifically, the co-activation of medial and lateral perforant pathways yields a greater enhancement than similar activation of the individual pathways alone ( $\mathrm{Mc}$ Naughton et al., 1978). Moreover, the threshold for obtaining enhancement appeared to be reduced when the two afferents were co-activated. In contrast, a recent report by Douglas et al. (1982) indicates that co-activation of the commissural inputs which terminate on the proximal portion of the same dendrites significantly attenuates the enhancement of perforant path responses. The present study sought to examine whether synaptic inputs to the stratum radiatum of CA1 exhibit interactive characteristics with respect to the induction and degree of long-term potentiation. In particular, the effect of coactivating adjacent afferents to the stratum radiatum was examined in the in vitro hippocampal slice preparation. Furthermore, the nonsynaptic co-activation of the postsynaptic pyramidal cells (in conjunction with synaptic activation of the stratum radiatum) was undertaken to examine the possible contribution of postsynaptic cellular discharge to the phenomenon of synaptic potentiation.

\section{Matcrials and Methods}

Slices from the dorsal (septal) aspect of the rat hippocampus were prepared as described in detail elsewhere (Spencer et al., 1976). Following a 1-hr period of equilibration, the combined Schaffer collateral and commissural inputs to CA1 were tested by placing stimulating and recording electrodes as depicted in Figure 1. The viability of the slices was tested according to previously published criteria (Lee et al., 1981). If the slices satisfied these criteria, then stimulation intensities were adjusted such that extracellularly recorded field potentials (Andersen et al., 1977) of 1.5 to $2.0 \mathrm{mV}$ were elicited. Since these pathways generate boutons en passage, synaptic activation of CA1 pyramidal cells can be achieved by stimulation of the Schaffer collateral and commissural pathways in an orthodromic (i.e., from the CA3 aspect of the slice; $\mathrm{OS} / \mathrm{C}$ ) or antidromic (i.e., from the subicular aspect; AS/C) direction (Dunwiddie and Lynch, 1978). 


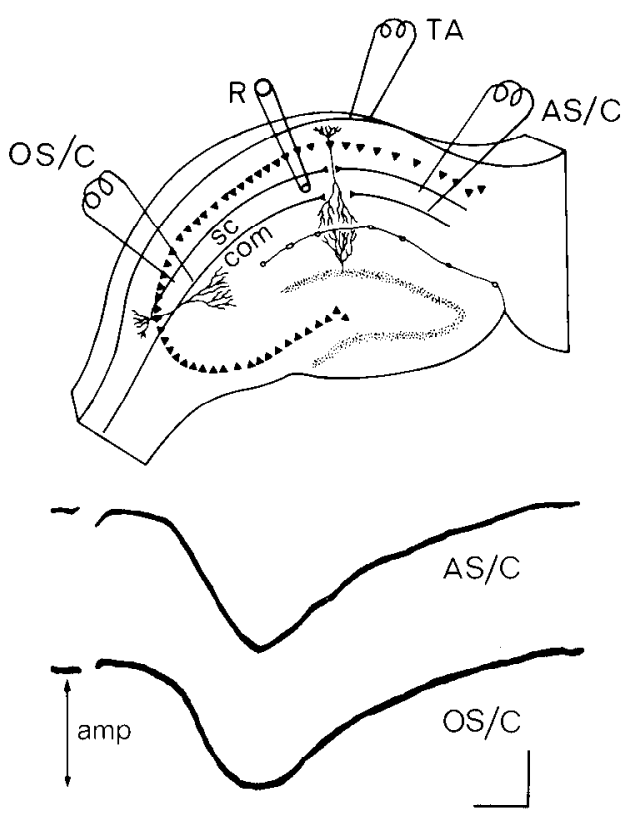

Figure 1. Above, the placements of stimulating and recording electrodes in the hippocampal slice. Since the Schaffer collateral and commissural afferents generate most of their boutons in an en passage fashion, synaptic activation of postsynaptic pyramidal cells in CA1 can be achieved by stimulating in either an "orthodromic" $(O S / C)$ or "antidromic" $(A S / C)$ direction. The extracellularly recorded responses from these stimulation placements are similar, and examples of oscilloscope traces (from the same slice) are shown below. In the OS/C-elicited response, the manner in which amplitude measurements were performed by a MINC 11 computer is shown. Calibration bars are $0.5 \mathrm{mV}$ and $5 \mathrm{msec}$. In certain experiments, a stimulating electrode was placed in the alveus $(T A)$. This stimulation site antidromically activates CA1 pyramidal cells. com, commissural afferents; $R$, recording electrode in the stratum radiatum; $s c$, Schaffer collateral afferents.

Responses were evoked at 5-sec intervals in the OS/C pathway, and the amplitude of every fourth potential was measured by a MINC 11 computer as shown in Figure 1. If a stable base line of responses was obtained over a 10- to 15-min test period, then repetitive, conditioning stimulation was delivered according to one of the following paradigms: (1) OS/C only, three bursts at 250 $\mathrm{sec}^{-1}$ for $200 \mathrm{msec}$; (2) OS/C and AS/C, three bursts at $250 \mathrm{sec}^{-1}$ for $200 \mathrm{msec}$; or (3) OS/C only with the duration of individual pulses increased by 10 -fold $(\mathrm{OS} / \mathrm{C} \times 10)$, three bursts at $250 \mathrm{sec}^{-1}$ for $200 \mathrm{msec}$. In another series of experiments, a stimulating electrode was placed in the OS/C pathway with a second stimulator located in the alvear region of CA1. The alvear stimulator (TA) primarily activates the axons of CA1 pyramidal cells, thus antidromically activating these neurons. The strength of TA stimulation was adjusted such that the amplitude of evoked responses recorded in the pyramidal cell layer was 2.0 to $2.5 \mathrm{mV}$. The TA stimulator was co-activated with the OS/C stimulator only during conditioning pulses, which were of similar rate and duration as those described above. Subsequent to each of the above conditioning paradigms, test stimuli were administered to the OS/C pathway at the intensity and rate utilized for the preconditioning base line responses. Responses in the
$\mathrm{OS} / \mathrm{C}$ pathway were followed for approximately $30 \mathrm{~min}$ after the application of conditioning stimuli. The amount of change in response amplitude was quantified at 5 and 20 min after conditioning as follows:

$$
\% \text { Change }=\left(\frac{A t-A b}{A b}\right) \times 100
$$

In this equation, "At" is the amplitude of the evoked response at either 5 or $20 \mathrm{~min}$ following conditioning stimulation, and " $A b$ " is the base line response amplitude prior to conditioning.

\section{Results}

Each of the conditioning paradigms which were employed resulted in an increase in the amplitude of the extracellularly recorded population EPSP (Table I). The magnitudes of these increases, however, varied according to the type of conditioning stimulation utilized. When only the orthodromic Schaffer/commissural stimulation electrode (OS/C) was repetitively activated (at normal stimulation strength, see above), 15 of 15 slices exhibited an increase in response amplitude $5 \mathrm{~min}$ following conditioning. However, by $20 \mathrm{~min}$ after the conditioning stimulation, only 5 of 15 slices were potentiated by more than $5 \%$ over their base line levels (Table I). Figure 2 shows two cases in which such conditioning stimuli were applied. This figure illustrates what seem to be two distinct forms of modification of the evoked response. In the first case (Fig. 2, upper graph), the increase in response amplitude is clearly decremental and reaches a preconditioning base line level within 10 to 15 min after high frequency activation. In contrast, the second type of increase (Fig. 2, lower graph) is observed to decrease for the first 5 to $10 \mathrm{~min}$, after which time the enhancement of the response appears to be relatively stable. The five slices that displayed an enhancement of more than $5 \%$ above base line levels exhibited this latter form of change. The other 10 slices in this group continuously declined to base line levels in a fashion similar to the slice shown in Figure 2, upper graph.

When the AS/C was co-activated (only during the conditioning bursts) with the OS/C, a greater increase in the OS/C evoked potential amplitude was obtained during subsequent test stimulation (Table I). At both 5 and 20 min postconditioning, the enhancement was significantly greater than that observed following $\mathrm{OS} / \mathrm{C}$ conditioning alone $(5 \mathrm{~min}, p<0.001 ; 20 \mathrm{~min}, p<0.01$,

\section{TABLE I}

Enhancement of evoked potentials of CA1

The enhancement of evoked potentials was quantified at 5 and 20 min following conditioning by calculating the percentage increase over preconditioning base line levels (see "Materials and Methods"). The values shown are means \pm SEM for each conditioning paradigm. The $n$ values refer to the number of slices which were examined for each condition.

\begin{tabular}{lccr}
\hline \multirow{2}{*}{$\begin{array}{l}\text { Stimulation } \\
\text { Paradigm }\end{array}$} & $n$ & \multicolumn{2}{c}{ Time Postconditioning } \\
\cline { 3 - 4 } & & 5 min & \multicolumn{1}{c}{$20 \mathrm{~min}$} \\
\hline $\mathrm{OS} / \mathrm{C}$ & 15 & $21.9 \pm 3.9$ & $7.3 \pm 3.1$ \\
$\mathrm{OS} / \mathrm{C}+\mathrm{AS} / \mathrm{C}$ & 13 & $33.5 \pm 5.0$ & $22.5 \pm 4.3$ \\
$\mathrm{OS} / \mathrm{C} \times 10$ & 11 & $65.9 \pm 8.6$ & $33.4 \pm 6.9$ \\
$\mathrm{OS} / \mathrm{C}+\mathrm{TA}$ & 9 & $16.1 \pm 3.9$ & $7.9 \pm 4.1$ \\
\hline
\end{tabular}



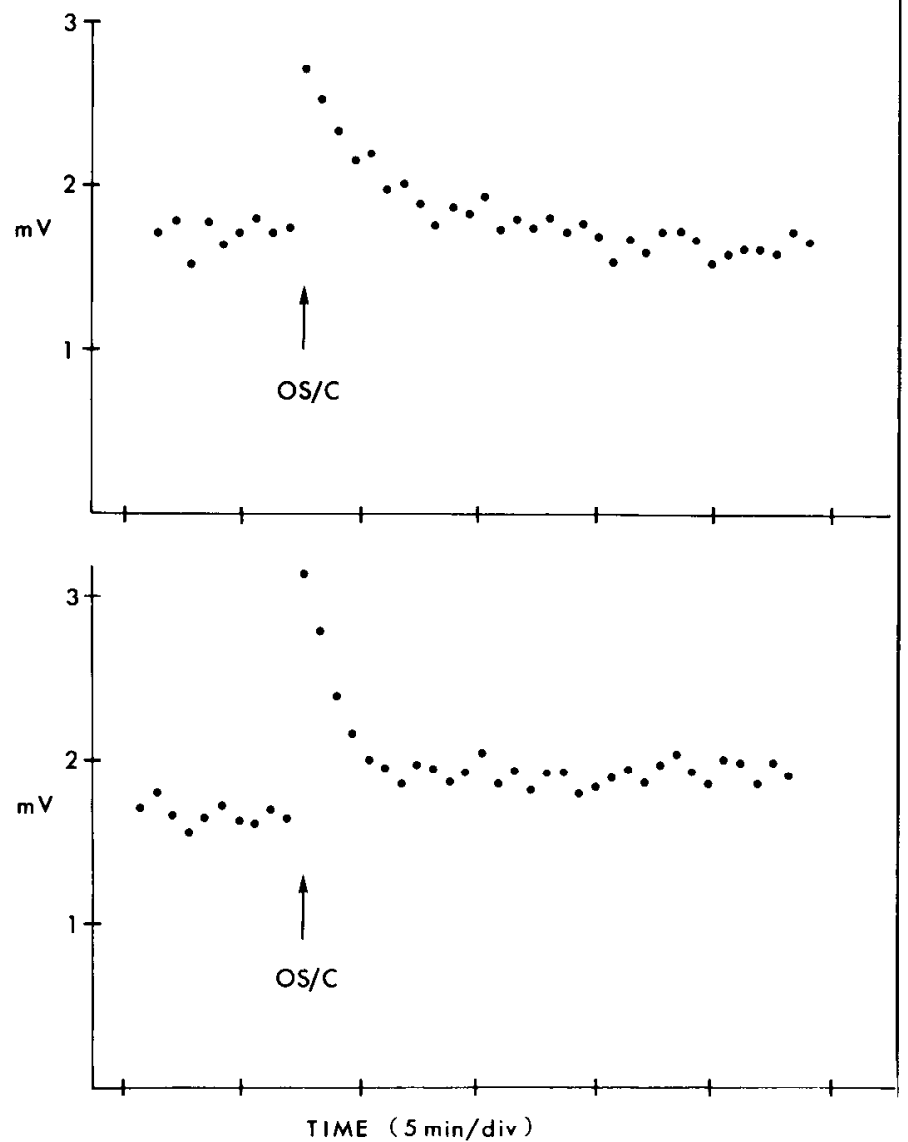

Figure 2. Time courses of the amplitudes of OS/C evoked responses in two experiments in which $\mathrm{OS} / \mathrm{C}$ conditioning was administered. Arrows indicate the points at which conditioning stimuli were delivered. The slice shown in the upper graph exhibited a transient increase in amplitude which returned to base line 5 to $10 \mathrm{~min}$ after conditioning. The lower graph shows another slice which remained potentiated for the duration of the experiment following OS/C conditioning.

Student's $t$-test, two-tailed). Five minutes after OS/C + $\mathrm{AS} / \mathrm{C}$ conditioning, all slices $(n=13)$ were potentiated. Moreover, 12 of the 13 slices were still potentiated by more than $5 \%$ at 20 min postconditioning. An example of the enhancement elicited by $\mathrm{OS} / \mathrm{C}+\mathrm{AS} / \mathrm{C}$ co-activation is shown in Figure 3, upper graph.

The third type of conditioning stimulation involved the activation of only the OS/C pathway, but with the duration of the individual stimulation pulses increased by 10 -fold only during the conditioning volley (OS/C $\times$ 10). The results of one such experiment are shown in Figure 3, lower graph. This type of stimulation resulted in the greatest degree of enhancement of all of the conditioning paradigms. The initial few responses following conditioning (which presumably reflect post-tetanic potentiation), as well as the measures taken at 5 and 20 min, were clearly greater than those obtained either with low intensity conditioning of $\mathrm{OS} / \mathrm{C}$ alone or with coactivation of $\mathrm{OS} / \mathrm{C}$ and $\mathrm{AS} / \mathrm{C}$. These changes were significantly greater at 5 and $20 \mathrm{~min}$ postconditioning when compared with any of the other conditions (all significance levels were $p<0.001$, except for the comparison of
$\mathrm{OS} / \mathrm{C}+\mathrm{AS} / \mathrm{C}$ versus $\mathrm{OS} / \mathrm{C} \times 10$ at $20 \mathrm{~min}$, which was $p$ $<0.02$ ).

The fourth class of conditioning stimulation involved the co-activation of the TA with the OS/C pathway. This stimulation paradigm resulted in a potentiation of OS/C responses which was virtually identical to that obtained with OS/C conditioning alone (Table I).

\section{Discussion}

The evidence derived from the present experiments demonstrates that afferents to the stratum radiatum of CA1 exhibit a form of cooperativity with respect to the induction and maintenance of sustained synaptic potentiation. The cooperative nature of inputs to the stratum radiatum of CA1 is supported by: (1) the greater degree of potentiation following co-active conditioning $\mathrm{OS} / \mathrm{C}$ and $\mathrm{AS} / \mathrm{C}$ pathways in comparison to activation of only the OS/C pathway and (2) the greater potentiation following conditioning of the OS/C pathway with a higher strength of conditioning stimulation. These observations are consistent with data from the dentate gyrus in which afferents from the entorhinal cortex exhibit a similar

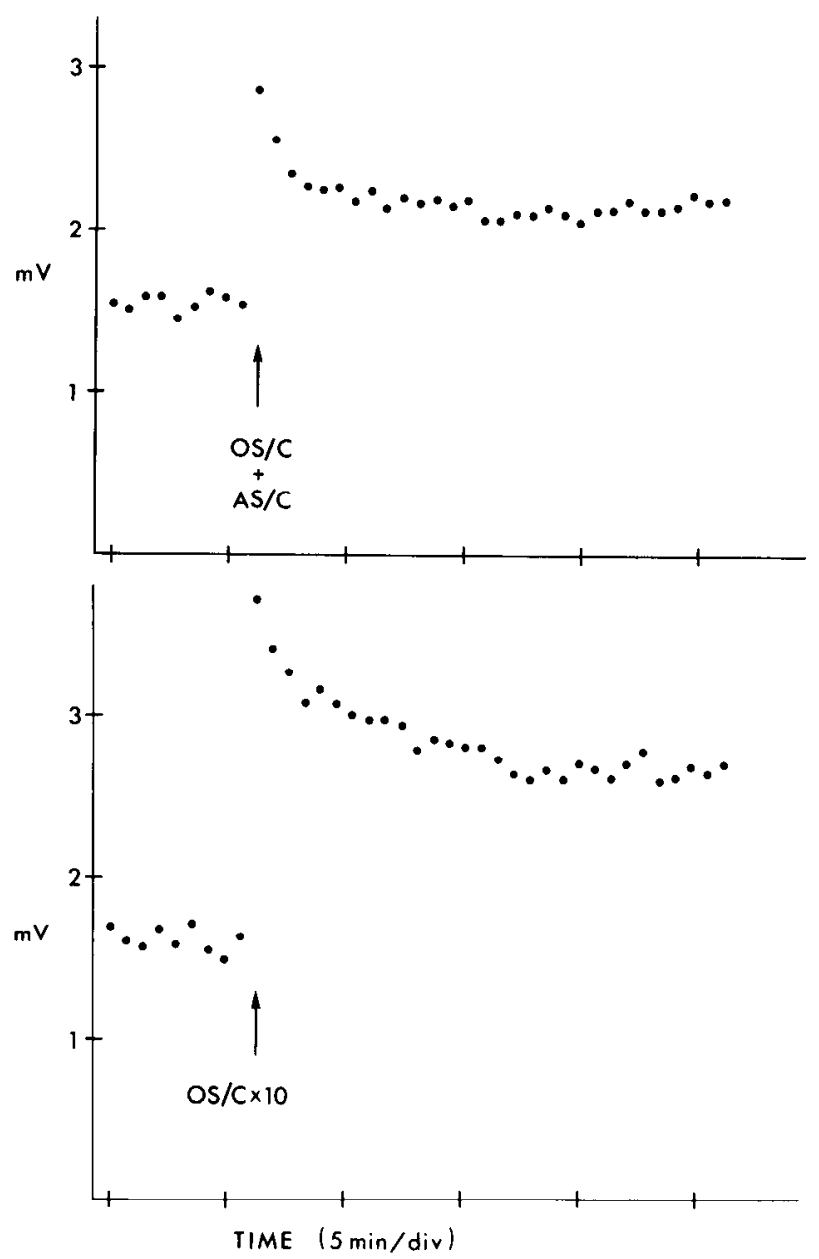

Figure 3. Time courses of the amplitudes of OS/C evoked potentials from two slices, one of which received OS/C + AS/ $\mathrm{C}$ conditioning (upper graph) and one which received OS/C $\times 10$ conditioning stimulation (lower graph). Both conditions elicited an increase in response amplitude greater than that observed with $\mathrm{OS} / \mathrm{C}$ conditioning alone. The $\mathrm{OS} / \mathrm{C} \times 10$ conditioning paradigm was the most effective of those tested. 
type of cooperativity for the induction of synaptic enhancement (McNaughton et al., 1978). Furthermore, the greater degree of potentiation in the present study does not seem to be due to a greater discharge of postsynaptic pyramidal cells. Nonsynaptic, antidromic co-activation of pyramidal cells did not enhance the degree of longterm potentiation which was obtained.

In the first series of experiments, synaptic activation of the same set of postsynaptic cells was achieved by stimulating either the subicular aspect of CA1 (AS/C) or the border between CA1 and CA3 (OS/C) in the stratum radiatum. While activating the same population of postsynaptic cells, the two stimulation sites seem to activate synaptic populations which do not significantly overlap; potentiation of responses from one stimulation site (either OS/C or AS/C) normally does not enhance the response to the other, nontetanized input (Dunwiddie and Lynch, 1978). In comparison to the potentiation elicited by stimulation of one of these sites, co-active conditioning of the two stimulation sites, utilizing the same stimulation parameters, resulted in a significantly greater potentiation of the individual pathways. It thus appears that the co-activation of adjacent populations of synapses results in a potentiation of the capacity to exhibit synaptic enhancement.

The second series of experiments utilized only a single stimulation site (i.e., OS/C); however, by altering the strength of the conditioning stimuli, the degree of synaptic co-activation could be modified. The primary observation from these experiments was that higherstrength conditioning stimulation induced a greater degree of potentiation. Presumably, the increased strength of conditioning stimulation resulted in a co-activation of a larger number of adjacent synapses. The resultant greater enhancement is consistent with the hypothesis that the amount of potentiation is related to the degree of co-activation of input to the CAl pyramidal cells. It is noteworthy that, in certain slices, the lowest strength of conditioning stimulation which was applied (i.e., a stimulation intensity which evoked a population EPSP of 1 $\mathrm{mV}$ amplitude; data not shown) was capable of enhancing subsequent responses. Such increases were usually small and normally decayed to a base line level within $60 \mathrm{~min}$. This observation does not support or refute the notion that a minimal degree of stimulation (i.e., co-activation) is required to elicit long-term potentiation. That is, it remains unclear whether a certain, minimum number of synaptic sites needs to be co-activated before long-term potentiation can be induced (Schwartzkroin and Wester, 1975; McNaughton et al., 1978).

The final series of experiments employed an antidromic, nonsynaptic conditioning (TA) of the postsynaptic pyramidal cells in conjunction with orthodromic, synaptic activation of the stratum radiatum afferents $(\mathrm{OS} / \mathrm{C})$. If an increase in the discharge of the postsynaptic cells during conditioning were related to the greater degree of long-term potentiation seen with the other forms of co-activation (i.e., with $\mathrm{OS} / \mathrm{C}+\mathrm{AS} / \mathrm{C}$ or OS/C $\times 10$ ), then the antidromic, co-active conditioning should have mimicked the other co-activation paradigms. This, however, did not occur; the resultant degree of potentiation was similar to that obtained with OS/C stimulation alone. Therefore, it seems that the greater amount of potentiation among co-activated, stratum radiatum afferents was due to an interaction in the region of synaptic termination rather than some effect of increased postsynaptic discharge during conditioning. The present studies cannot resolve whether the observed interactions occur at a presynaptic, postsynaptic, or perhaps glial sile(s). Furthermore, because all of the recordings were taken in the dendritic zone (where the afferents terminate), it is unclear whether the discharge of pyramidal cells is somehow differentially modified by the various conditioning paradigms. For instance, a direct effect on spike discharge characteristics by the conditioning stimuli would not be resolved with the dendritic recordings. While these additional issues await clarification, the present results clearly demonstrate the existence of an associative (cooperative) capacity for the induction and maintenance of synaptic potentiation of stratum radiatum afferents and that this interaction occurs in the local region of the synapse.

\section{References}

Alger, B. E., and T. J. Teyler (1976) Long-term and short-term plasticity in the CA1, CA3 and dentate regions of the rat hippocampal slice. Brain Res. 110: 463-480.

Andersen, P., S. H. Sundberg, $O$. Sveen, and H. Wigstrom (1977) Specific long-lasting potentiation of synaptic transmission in hippocampal slices. Nature 266: 736-737.

Bliss, T. V. P., and A. R. Gardner-Medwin (1973) Long-lasting potentiation of synaptic transmission in the dentate area of the unanesthetized rabbit following stimulation of the perforant path. J. Physiol. (Lond.) 232: 357-374.

Bliss, T. V. P., and T. Lomo (1973) Long-lasting potentiation of synaptic transmission in the dentate area of the anaesthetized rabbit following stimulation of the perforant path. J. Physiol. (Lond.) 232: 331-356.

Douglas, R. M., and G. V. Goddard (1975) Long-term potentiation of the perforant path-granule cell synapse in the rat hippocampus. Brain Res. 86: 205-215.

Douglas, R. M., G. V. Goddard, and M. Riives (1982) Inhibitory modulation of long-term potentiation: Evidence for a postsynaptic locus of control. Brain Res. 240: 259-272.

Dunwiddie, T., and G. Lynch (1978) Long-term potentiation and depression of synaptic responses in the rat hippocampus: Localization and frequency dependency. J. Physiol. (Lond.) 276: 353-367.

Lee, K. (1982) Sustained enhancement of evoked potentials following brief, high-frequency stimulation of the cerebral cortex in vitro. Brain Res. 239: 617-623.

Lee, K., F. Schottler, M. Oliver, and G. Lynch (1981) Electron microscopic studies of brain slices: The effects of high-frequency stimulation on dendritic ultrastructure. In Electrophysiology of Isolated Mammalian CNS Preparations, G. A. Kerkut and H. V. Wheal, eds., pp. 189-211, Academic Press, London.

MeNaughton, B. L. (1982) Long-term synaptic enhancement and short-term potentiation in rat fascia dentata act through different mechanisms. J. Physiol. (Lond.) 324: 249-262.

McNaughton, B. L., R. M. Douglas, and G. V. Goddard (1978) Synaptic enhancement in fascia dentata: Cooperativity among coactive afferents. Brain Res. 157: 277-293.

Schwartzkroin, P. A., and K. Wester (1975) Long-lasting facilitation of a synaptic potential following tetanization in the in vitro hippocampal slice. Brain Res. 89: 107-119.

Spencer, H. J., V. Gribkoff, C. Cotman, and G. Lynch (1976) GDEE antagonism of iontophoretic amino acid excitations in the intact hippocampus and in the in vitro hippocampal slice preparation. Brain Res. 105: 471-481. 